\title{
Violencia de género y riesgo de feminicidio en alumnas de universidades públicas y privadas de Lima Metropolitana
}

\author{
Gender violence and risk of feminicide in pupils from public and private \\ universities in Lima Metropolitana
}

\author{
Carlos Velásquez Centeno ${ }^{1}$, Alex Grajeda Montalvo ${ }^{2}$, William Montgomery \\ Urday ${ }^{3}$, Víctor Montero López ${ }^{4}$, Ricardo Pomalaya Verastegui ${ }^{5}$, Kelly Pampa \\ Luque ${ }^{6}$ y Susy Flores Guerra ${ }^{7}$ \\ Universidad Nacional Mayor de San Marcos
}

\author{
José Vallejos Saldarriaga ${ }^{8}$ \\ Universidad Peruana Cayetano Heredia \\ Susana Cabrera Echegaray ${ }^{9}$ \\ Universidad Privada del Norte \\ David Zamudio Flores ${ }^{10}$ \\ Universidad Continental
}

Recibido: $18-09-20$

Aceptado: $16-11-20$

Publicado: $21-12-20$

1 Docente, Universidad Nacional Mayor de San Marcos. Lima, Perú.

Autor para correspondencia: cvelasquezc@unmsm.edu.pe ORCID: https://orcid.org/0000-0002-7266-4012

2 Docente, Universidad Nacional Mayor de San Marcos. Lima, Perú.

E-mail: agrajedam@unmsm.edu.pe ORCID: https://orcid.org/0000-0001-5972-2639

3 Docente, Universidad Nacional Mayor de San Marcos. Lima, Perú. E-mail: wmontgomeryu@unmsm.edu.pe, avidolector@yahoo.es ORCID: https://orcid.org/0000-0001-9518-3329

4 Docente, Universidad Nacional Mayor de San Marcos. Lima, Perú.

E-mail: vmonterol@unmsm.edu.pe ORCID: https://orcid.org/0000-0002-9968-4160

5 Docente, Universidad Nacional Mayor de San Marcos. Lima, Perú.

E-mail: rpomalayav@unmsm.edu.pe ORCID: https://orcid.org/0000-0002-3021-6895

6 Estudiante de pregrado de la Facultad de Psicología, Universidad Nacional Mayor de San Marcos. Lima, Perú.

E-mail: kely.palpa@unmsm.edu.pe ORCID: https://orcid.org/0000-0002-2212-8828

7 Estudiante de pregrado de la Facultad de Psicología, Universidad Nacional Mayor de San Marcos. Lima, Perú.

E-mail: Susy.flores@unmsm.edu.pe ORCID: https://orcid.org/0000-0002-0630-9426

8 Docente Universidad Peruana Cayetano Heredia y Universidad César Vallejo.

E-mail: jvallejoss@ucv.edu.pe ORCID: https://orcid.org/0000-0001-9653-1428

9 Docente en la Facultad de Humanidades de la Universidad Privada del Norte.

E-mail: susanahayd@hotmail.com ORCID: https://orcid.org/0000-0001-6874-1372

10 Docente en las Facultades de Humanidades y Ciencias de la Salud de la Universidad Continental.

E-mail: david.zamudio@unmsm.edu.pe ORCID: https://orcid.org/0000-0002-4977-1039

(C) Los autores. Este artículo es publicado por la Revista de Investigación en Psicología de la Facultad de Psicología, Universidad Nacional Mayor de San Marcos. Este es un artículo de acceso abierto, distribuido bajo los términos de la licencia Creative Commons Atribucion - No Comercia_Compartir Igual 4.0 Internacional. (http://creativecommons.org/licenses/by-nc-sa/4.0/) que permite el uso no comercial, distribución y reproducción en cualquier medio, siempre que la obra original sea debidamente citada. 


\title{
Resumen
}

El objetivo del estudio es evaluar la relación entre las actitudes hacia la violencia de género y el extremo riesgo de la pareja de ser víctima de violencia o feminicidio, con estudiantes de género femenino en universidades públicas y privadas de Lima Metropolitana, siendo los instrumentos a utilizar la Escala de Actitud Hacia la Violencia de Género y la Escala de Evaluación del Riesgo. La aplicación de los instrumentos se efectúa sobre una muestra de estudiantes de universidades públicas y privadas de las áreas de Ciencias de la Salud. Los resultados generales indican que a mayor actitud de rechazo a la violencia, existe menor experimentación de violencia; las mujeres de universidades privadas experimentan mayor violencia física, sexual y económica, y menor rechazo a la violencia de género; las jóvenes muestran un mayor rechazo a la violencia que las adultas; las mujeres que tienen pareja experimentan mayor violencia psicológica y económica; y las personas auto-empleadas son las que consideran sufrir mayor violencia en todos sus tipos a comparación de aquellas empleadas y desempleadas.

Palabras clave: Violencia de género; riesgo de violencia extrema sobre la pareja; feminicidio; violencia hacia la mujer; Violencia de género en la universidad.

\begin{abstract}
The objective of the study is to evaluate the relationship between attitudes towards gender violence and the risk of extreme violence towards the couple or feminicide in female students of public and private universities of Metropolitan Lima, being the instruments to use the Attitude Scale Towards Violence Gender and Risk Assessment Scale. The instruments are applied on a sample of students from public and private universities in the areas of Health Sciences. The general results indicate that the greater the attitude of rejection of violence, there is less experimentation of violence; women from private universities experience greater physical, sexual and economic violence, and less rejection of gender violence; young people show a greater rejection of violence than adults; women who have a partner experience greater psychological and economic violence; and self-employed people are those who consider suffering the greatest violence in all types compared to those employed and unemployed.
\end{abstract}

Keywords: Gender violence; risk of extreme violence against the couple; feminicide; violence towards women; Gender violence in the university.

Según la Organización Mundial de la Salud (OMS, 2002) la violencia en general constituye un "uso deliberado de la fuerza física o el poder, ya sea en grado de amenaza o efectivo, contra uno mismo, otra persona o un grupo o comunidad, que cause o tenga muchas probabilidades de causar lesiones, muerte, daños psicológicos, trastornos del desarrollo o privaciones" (p. 5). En este marco, las concepciones que actualmente se etiquetan como "violencia de género" (o violencia basada en el género ${ }^{11}$ y "feminicidio" 12 tienen que ver, obviamente, con formas de

11 Es bueno aclarar de entrada la diferencia entre "sexo" y "genero" de acuerdo con estimaciones modernas. El sexo tiene un carácter fundamentalmente biológico, mientras que el género involucra aspectos de "masculinidad" y "feminidad" que, si bien tienen un asidero biológico, lo rebasan, constituyendo una experiencia personal de autoidentidad sexual no necesariamente coincidente con el sexo biológico (Gómez, 2013).

12 Concepto introducido en 1976 por la Psicóloga social y feminista sudafricana Diana Russell, impulsora del Primer Tribunal Internacional de Crímenes contra Mujeres. Lo define como un crimen de odio que implica el asesinato de una mujer por el hecho de ser mujer (Russell \& Harmes, 2006). 
agresión contra la mujer que se han ido extendiendo en la sociedad contemporánea hasta poner el problema en la mira de sectores profesionales, que lo ven desde un punto de vista no solo social, sino sobre todo sanitario. Cabe señalar que la misma OMS (2014) califica la violencia contra la mujer como "un problema importante de salud pública y de derechos humanos" (p. 3), lo cual propicia que muchos gobiernos del orbe incluyan políticas de prevención respecto al punto.

La violencia interpersonal, en su sentido más amplio, puede ser connotada de dos maneras: una como "violencia-agresión" y otra como "violencia-castigo", utilizando una clasificación realizada en el año 2006 por los investigadores argentinos R. Perrone y M. Nannini (citados por Matamoros, 2014, pp. 35-36). De acuerdo con este razonamiento, la violencia-agresión implica simetría en la relación de contacto, es decir dos personas en igualdad de condiciones que se ofenden o agreden mutuamente. En cambio la violencia-castigo se ejerce en una relación de complementareidad no igualitaria, donde una de las partes se halla en una situación de poder y la otra de sumisión. Es, pues, el contexto de violencia-castigo en que predomina en relaciones de pareja heterosexuales - reguladas por una idiosincrasia y legislación patriarcales milenarias de sobra conocidas, donde el género femenino está en clara desventaja (Arcila, 2014, Abramovich, 2014)-, que reflejan en su faceta más extrema el uso de prácticas de esclavización, violación, humillación, lesiones corporales e incluso el asesinato, con cierto grado de impunidad.

Por ello puede definirse la violencia de género como "un conjunto de actitudes, vivencias y comportamientos violentos que producen daño, malestar y sufrimiento a la víctima ya su entorno más próximo y vulnerable (hijos pequeños, padres mayores, familiares discapacitados, etc.)" (Zurita, 2014; p. 27). De otro lado, la Organización de las Naciones Unidas (ONU), en una declaración de su asamblea, califica la violencia contra la mujer como "todo acto de violencia basado en la pertenencia al sexo femenino que tenga o pueda tener como resultado un daño o sufrimiento físico, sexual o psicológico para la mujer, así como las amenazas de tales actos, la coacción o la privación arbitraria de la libertad, tanto si se producen en la vida pública como en la vida privada" (Organización de las Naciones Unidas, 1993 $)^{13}$. A su vez, la Convención Interamericana para Prevenir, Sancionar y Erradicar la Violencia Contra la Mujer (Convención de Belem do Pará, 1994, citada por el Ministerio de la Mujer y Poblaciones Vulnerables del Perú [MIMP, 2016, p. 11]) le añade "«basada en género» destacando que gran parte de esta violencia se origina en una estructura social discriminante con las mujeres solo por el hecho

13 Esto ha sido tomado parcialmente por la Ley 30364 en el Perú: "Ley Para Prevenir, Sancionar y Erradicar la Violencia contra las Mujeres y los Integrantes del Grupo Familiar”, que define así la violencia contra las mujeres: "Cualquier acción o conducta que les causa muerte, daño o sufrimiento físico, sexual o psicológico por su condición de tales, tanto en el ámbito público como en el privado" (citado en línea por el Observatorio Nacional de la Violencia contra las Mujeres y los Integrantes del Grupo Familiar, 2019). 
de ser mujeres, desvalorizando lo femenino, y entre hombres y mujeres construir desigualdades sociales".

Una muestra de la histórica discriminación idiosincrásica y legal que ha sido mencionada, es el mayor riesgo de exclusión social para el género femenino en diversos campos. Por ejemplo, hasta hace muy poco, los "delitos contra el honor" eran concebidos de manera desigual para el hombre y para la mujer. Se consideraba delitos contra el honor masculino la infidelidad de la mujer (dándole derecho al varón para "limpiar su honor" el cobrar con la vida de su mujer y del amante de esta, y pérdida de honor para la mujer el ser violada o desvirgada fuera del matrimonio (Arcila, 2014). Además, no es necesario profundizar mucho para darse cuenta de que: "Como grupo, las mujeres tienen menos oportunidad de ser encontradas en la esfera pública que los hombres, y cuando lo están, tienen menos recursos a su disposición" (Connel \& Pearce, 2015; p. 33).

Como señala Zurita (2014), en la maldición bíblica a Eva ubicada en el Génesis 3:1 se puede encontrar la referencia más antigua respecto a la desigualdad entre el hombre y la mujer (“tu deseo será para tu marido, y él se enseñoreará de ti”). Algo parecido se encuentra siglos después en el Corán ("en virtud de la preferencia que Alá ha dado a unos más que a otros, los hombres tienen autoridad sobre las mujeres [...] ¡Reprended a aquéllas de quienes temáis que se rebelen, solas en el lecho, pegadles!": Sura 4.34). En las antiguas civilizaciones griega, romana y árabe, la mujer muchas veces era reducida a la categoría de propiedad, continuando dicha situación durante toda la Edad Media. Solo en el siglo XVII esta discriminación y violencia contra la mujer comienzan a ser cuestionadas, consiguiéndose poco a poco aumentar el caudal de derechos conquistados durante el siglo XX. Así por ejemplo, en 1928 la Organización de Estados Americanos (OEA), funda la Comisión Interamericana de Mujeres (CIM); en los años 50s la OIT en su objetivo de defensa internacional del trabajo, reclama en su discurso la igualdad de remuneración en el trabajo entre los géneros; y la ONU convoca a sus miembros a abolir seculares costumbres y leyes discriminativas. En el último tercio del siglo XX y en el actual siglo XXI, salvo unos pocos países dominados por ideologías religiosas fundamentalistas, la mayoría está de acuerdo con el cambio de políticas de género. En nuestro país también se suscribieron acuerdos promovidos por la Convención Interamericana para Prevenir, Sancionar y Erradicar la Violencia Contra la Mujer (antes citada) para recopilar información sobre sus "causas, consecuencias y frecuencia", a fin de adoptar medidas correctivas (MIMP, 2016). Incluso se ha articulado un organismo denominado "Observatorio Nacional de la Violencia contra las Mujeres y los Integrantes del Grupo Familiar", encargado de hacer durante el período 2016-2021, el seguimiento al Plan Nacional Contra la Violencia de Género. Sin embargo, a pesar de esos avances, todavía existen tremendas desigualdades. A este respecto hay que hacer la atingencia de que para superarlas es bueno adoptar un camino moderado: actitudes fanáticas como el machismo (y su contraparte 
extrema: el feminismo) distorsionarían el carácter de tales avances, amenazando con dirigir el proceso de reivindicación hacia otras fronteras.

Como se ha visto, la violencia de género está afincada en patrones de orden ontogenético -es decir, de carácter aprendido. Es un proceso de modelamiento sociocultural que "exige" desempeñar papeles de acuerdo con modelos diferentes en cada uno de los géneros: el femenino debe manifestar habilidades de cuidado del hogar y de pasividad sexual, y el masculino de proactividad sexual y financiera. Esto está confirmado no solo por la observación convencional, sino por múltiples estudios a nivel mundial en diferentes continentes, como los realizados por Eastwick, Eagly \& Glick en 2006 (citados por Cunha, Alves, Gushi, Farias, Murrins, Moraes, Viera, Ferreira \& Barreto (2013; p. 118). El desempeño de esos papeles culturales pone automáticamente en posición de autoridad al "proveedor financiero", empoderando sus actos como "jefe del hogar", y por tanto, como el creador, intérprete y censor de las reglas que deben cumplirse dentro de la convivencia de la pareja. Muchas veces, la violencia de género y el feminicidio vienen a ser la consecuencia de una interpretación rígida de tales reglas, en relación además con un estado afectivo particular personal del perpetrador del delito. Hay, entonces, posibles factores de riesgo asociados a estos actos que pueden agruparse a niveles individual y social (véase figura 1):

\begin{tabular}{ll}
\hline & Nivel individual \\
\hline De ser perpetrador & De ser víctima \\
- Desempleo. & Embarazo. \\
- Propiedad de armas de fuego. & Abuso durante el embarazo. \\
- Amenazas de matar con un arma. & Maltrato infligido anteriormente por el perpetrador, \\
- Imponer a la pareja tener relaciones sexuales. & especialmente maltrato grave en los 30 días prece- \\
- Abuso extremo del alcohol y uso de drogas & dentes, y abuso cada vez más frecuente. \\
ilegales. & Un niño nacido de una relación anterior (que no sea \\
- Dificultades de salud mental. & hijo biológico del delincuente) \\
pareja. & - Separación de la pareja. \\
\hline
\end{tabular}

\begin{tabular}{l} 
Nivel social / estructural \\
\hline - Trato desigual por motivos de género, incluso exiguo número de personas de género femenino entre las auto- \\
ridades del gobierno que han sido elegidos. \\
- Disminuciones significativa de gastos sociales gubernamentales en sectores como educación y salud (de \\
gastos de consumo final gubernamentales).
\end{tabular}

Figura 1. Posibles factores de riesgo individuales y sociales relacionados con el femicidio.

Fuente: Adaptado de OMS (2014). Respuesta a la violencia de pareja y a la violencia sexual contra las mujeres. Directrices de la OMS para la práctica clínica y las políticas. (p. 4).

Como se ve en el figura 1, si bien la violencia de género puede provenir en principio de cualquiera de los dos géneros, el feminicidio tiene características especiales al ser cometido en su mayoría por una pareja actual o anterior de la víctima. Existe mayor probabilidad de que una mujer sea agredida o asesinada por su (ex)pareja 
que por un extraño, cosa que no sucede en la misma dimensión en el caso de los hombres (Hernández, Raguz, Morales y Burga, 2018). Igualmente, hay presencia de maltrato recurrente en la forma de agresiones tales como amenazas o intimidación en condiciones de sumisión, sean sexuales o económicas. Esta agresión puede ser reactiva o proactiva (Matamoros, 2014), reactiva si tiende a producirse como respuesta de enfado o frustración a algún tipo de amenaza o provocación real o percibida, y proactiva si se hace planificadamente para satisfacer un deseo de causar daño para lograr satisfacción o conseguir un objetivo.

Pero, como señala Bandura (1984): "Las personas no nacen con repertorios prefabricados de conducta agresiva, deben aprenderlos de alguna manera" ( $p$. 310); y además esos repertorios violentos son mantenidos por ciertos mecanismos de interacción entre el individuo y su entorno. Por ello, su análisis del aprendizaje social de la agresión es pertinente en este sentido (figura 2).

\begin{tabular}{lll}
\hline \multicolumn{1}{c}{ Origen } & \multicolumn{1}{c}{ Instigadores } & \multicolumn{1}{c}{ Reforzamiento } \\
\hline - Aprendizaje por observación. & - Modelamiento. & - Reforzamiento externo. \\
- Ejecución reforzada. & - Tratamiento aversivo. & - Reforzamiento vicario. \\
- Determinantes estructurales. & - Móviles de incentivo. & Autorreforzamiento. \\
& - Control instruccional. & \\
& - Control simbólico y grotesco. & \\
\hline
\end{tabular}

Figura 2. Análisis del aprendizaje social de la agresión.

Fuente: Adaptado de Bandura (1984, p. 311).

La figura 2 evidencia posibles determinantes y consecuencias perpetuadoras del comportamiento violento o agresivo, en una explicación respaldada por abundante acopio experimental. En la primera columna se ven influencias de origen que pasan por el aprendizaje por observación (derivado del mal ejemplo familiar, social y mediático), la ejecución reforzada (acciones seguidas de aprobación o consecusión de objetivos) y determinantes estructurales (entornos subculturales y de privación). En la segunda columna están los posibles instigadores, es decir los que activan y canalizan los patrones agresivos deshinibiendo, facilitando o acrecentando el estímulo; o también respuestas a asaltos físicos, amenazas e insultos, reducción adversa del reforzamiento, frustración; y a veces anticipación de consecuencias, seguimiento de sugerencias u órdenes, así como convicciones de grandiosidad. En la tercera columna se aprecian clases de reforzamiento que mantienen o incrementan la agresión, como las recompensas tangibles y de estatus o el alivio de tratamiento aversivo; por otra parte recompensa y castigo observados; y finalmente dentro del autorreforzamiento acciones o pensamientos de autocastigo, autorrecompensa, neutralización del autocastigo, justificación moral, difusión de la responsabilidad, deshumanización de la víctima, atribución de culpa a las víctimas, y malinterpretación de consecuencias. 
Se supone que una vez aprendido el comportamiento en mención, hay mecanismos motivacionales que conforman lo que se llama una "actitud", disparando respuestas verbales y motoras que incluyen conducta cruel, oposicionista, burlona y otras afines, frente al objeto del rechazo actitudinal.

En este trance, es necesario que las investigaciones contribuyan a seguir esclareciendo elementos de origen, instigadores y reforzadores que incrementan tanto el riesgo de victimización por feminicidio, como el de que aparezcan perpetradores, con el fin de fortalecer estrategias de prevención eficaces. Esto es tanto más urgente cuanto en el Perú actual todavía las cifras de mujeres asesinadas siguen registrando índices superiores cada año (América Noticias, 27/12/2019). Según la Encuesta Demográfica y de Salud Familiar (ENDES), realizada en 2014 (citada por el MIMP, 2016, p. 20), hay factores de riesgo referentes a violencia física y sexual en relación con la edad de las mujeres peruanas: las más violentadas son las de edad avanzada. Asimismo, mujeres que se han divorciado, separado o son viudas presentan mayor violencia; y, en relación al nivel educativo, las mujeres con nivel secundario son más víctimas de violencia física. La violencia sexual está, también evidenciada entre mujeres que no tienen nivel educativo. La violencia con peligro de feminicidio en el Perú comprende a quienes en la encuesta del ENDES señalaron “en los últimos 12 meses su pareja (1) la estranguló o quemó, (2) atacó con cuchillo, pistola u otra arma o (3) amenazó con atacarla con cuchillo, pistola u otra arma" (Hernández, 2019).

Por todo lo expuesto, el objetivo de la presente investigación es evaluar la relación entre la violencia de género y la probabilidad de peligro de violencia máxima hacia la pareja en alumnos de universidades públicas y privadas de la región de Lima Metropolitana. A nivel teórico, el presente estudio busca entender mejor los problemas psicológicos que presentan los alumnos universitarios en su vida de pareja; así como a su vez, encontrar y analizar las posibles vinculaciones que puedan darse entre dichos fenómenos. A nivel práctico, los descubrimientos permitirán desarrollar acciones en torno a los comportamientos de violencia de género y el peligro de feminicidio en la pareja, priorizando los que sean más probables, y la topografía predominante de agresión hacia la pareja que exhiben los estudiantes universitarios. Asimismo, a un nivel tecnológico se intenta verificar la utilidad de las escalas de actitud hacia la violencia de género de Mateos (2011), y la de evaluación del riesgo de Hernández, et al. (2018), como instrumentos diagnósticos útiles para el establecimiento de factores protectores y de riesgo en las relaciones de pareja de los estudiantes universitarios.

Se espera que este estudio contribuya al acopiamiento de información actualizada sobre la violencia de género y la probabilidad de riesgo de violencia extrema (o feminicidio) hacia la pareja, y con ello contar con medidas eficaces en torno al problema abordado. 
Antecedentes de investigación nacional e internacional

Dentro de los antecedentes de investigación nacionales está la de Pacheco (2015), que busca determinar si existe relación entre la actitud hacia la violencia contra la mujer en la relación de pareja y el clima social familiar con sus dimensiones de "relación", "estabilidad", y "desarrollo", en adolescentes de cuarto año de secundaria. En dicha indagación se aplica una escala de actitud hacia la violencia contra la mujer en la relación de pareja y una escala de clima social familiar. Sus resultados no muestran correlación estadísticamente significativa entre estas variables. También Quispe et al. (2018) investigan sobre violencia contra la mujer y feminicidio, procurando determinar la tasa bruta de semejantes incidencias por año y por territorio, así como el riesgo de ocurrencia por territorio, año, mes, vínculo, escenario y área. Al analizar los resultados en el periodo 2009-2015, concluyeron que la incidencia de violencia extrema contra la mujer se incrementa; el peligro de feminicidio aumenta en el mes de noviembre, en los espacios rural y urbanomarginal, en un espacio no íntimo y cuando la violencia extrema contra la mujer no es efectuada por la pareja o ex-pareja.

Montenegro (2018) realizó una revisión del estado del arte de las principales peculiaridades de casos de feminicidio ocurridos en el Perú, usando el método de consulta o investigación bibliográfica, empleando las variadas publicaciones sobre la historia de vida como método de investigación. Comprende como feminicidio al crimen contra la mujer efectuado por un hombre, sea su pareja o expareja. El grupo etario donde se ubican, la víctima y el victimario, es de 18 a 34 años; la zona donde ocurrió el mayor porcentaje de feminicidios (59,7\%) es el interior de la casa donde viven o han vivido los cónyuges; la asfixia o el estrangulamiento es la forma más común de asesinar a la mujer (30,1\%); y de los casos sucedidos, el tipo feminicidio íntimo, es el que prevalece (80\%). Se hallaron como principales causas, las prácticas culturales que conceptúan a la mujer como un ser subordinado al hombre, en donde el que tiene el poder es el hombre, se evidencia así la tendencia ascendente del feminicidio en el Perú.

Otra investigación de relevancia es la de Hernández, et al (2018) sobre determinantes y evaluación del riesgo de feminicidio, en la cual constatan en primer lugar que aquel, así como su intento, son problemas poco explorados y comprendidos, incluso fuera del Perú. La exigua y restringida investigación en nuestro país sobre "violencia con riesgo de feminicidio" (como la llaman), contrasta con la premura de generar evidencia para canalizar las políticas públicas que buscan la reducción de la violencia contra las mujeres. Es así que, lo que se ha producido a la actualidad ha preferido el enfoque descriptivo o legal, siendo pocas las investigaciones multicausales que buscan asociar la incidencia de feminicidio con fenómenos económicos y sociales gravitantes. Las explicaciones esbozadas descansn sobre temas feministas de control, dominación y patriarcado, sin que estén respaldadas por la evidencia empírica y los progresos en las ciencias sociales. La 
evaluación de efectos contextuales efectuada por estos investigadores deja en claro ciertos aspectos de la presencia de instituciones sobre el riesgo de feminicidio: así, al par que el mayor número de policías, aumentó la variabilidad de este riesgo, la presencia de un Centro de Emergencia Mujer lo redujo. Asimismo, es evidente que la violencia con riesgo de feminicidio afecta gravemente en la salud mental y física ligadas a la depresión e hipertensión de las mujeres. A su vez, la violencia con riesgo de feminicidio promueve el incremento del consumo de alcohol y cigarros. El efecto sobre la salud física de los hijos e hijas se observa en el aumento de la incidencia de diarreas, tos y fiebre.

Siguiendo con este recuento, Aliaga y Figueroa (2019) realizaron una pesquisa cuyo principal objetivo era examinar la correlación entre violencia de pareja hacia la mujer y actitudes sexistas en universitarios, así como identificar el tipo de actitudes sexistas en cuanto a las tipologías de violencia, la agresión psicológica se presenta con más frecuencia que las demás. Se analizó también de acuerdo a si eran hombres o mujeres, ambos mostraban correlación entre las dos variables estudiadas. Por último, con respecto a las zonas, la zona urbana presenta con más frecuencia que las demás. Se analizó también de acuerdo al sexo entre hombres y mujeres, ambos presentan correlación entre las dos variables estudiadas. Por último, con respecto a las zonas, la zona urbana presenta una correlación directa positiva; por el contrario, la zona rural se muestra una correlación inversa no significativa.

También en nuestro medio Hernández (2019) hace notar que el Estado no debe contar las tentativas de feminicidio como si fuera un termómetro de violencia, porque el Ministerio Público (MP) solo contabiliza las tentativas de feminicidio en las cuales un fiscal formaliza una denuncia. Por eso las fuentes no coinciden en número ni en tendencia, y no se pueden contabilizar correctamente las tentativas de feminicidio.

Finalmente Quintana, Grajeda, Malaver, Medina, Montgomery y Ruiz (2019) usando redes semánticas estudian la presencia de estereotipos de género y el fenómeno de la violencia encubierta en parejas residentes en el populoso distrito limeño de San Martín de Porres. Entre sus resultados destaca que hombres y mujeres no presentan diferencias significativas en violencia encubierta y asimismo, sus estereotipos de género son muy particulares y alejados de los tradicionales.

Dentro de los antecedentes de investigación en el extranjero, Fernández (2013) de la Universidad de Oviedo, trata sobre "riesgo de feminicidio de género en situaciones de ruptura de la relación de pareja". Aquí señala cómo en relaciones de pareja caracterizadas por una situación de intenso dominio, el anuncio o la formulación de una decisión de ruptura en la relación de pareja, es actualmente la principal fuente o factor desencadenante de feminicidio. Este factor de riesgo es mayor que el de la denuncia policial o judicial de maltrato y, por tanto ambas, denuncia y separación, resultan ser parcialmente independientes entre 
sí. Lo paradójico es que mientras la denuncia del acto violento activa un sistema de medidas de protección física de la víctima, en cambio la separación no. Eso constituye un descuido de las autoridades que genera una situación de máximo riesgo y desprotección de la mujer.

Finalmente Rodríguez-Miñón (2016) da importantes pistas desde una perspectiva ecológica, acerca de aquellos factores de riesgo que convierten a algunos grupos femeninos en más susceptibles a sufrir violencia de género en España. Concluye que las inequidades de género son más acusadas en los ámbitos pequeños o rurales, que en los urbanos, "con el añadido de que además en estos últimos existen menos recursos especializados en violencia de género y recursos para la conciliación de la vida familiar y laboral, lo que principalmente afecta a las mujeres" (p. 44), por lo que propone centrar allí la atención preventiva. Como lo puntualizan Pueyo, López y Álvarez (2008): "Una de las formas de intervención preventiva... es la predicción de la violencia futura ya que de esta forma se pueden evitar nuevas agresiones e incluso la muerte de la mujer" (p. 115).

\section{METODOLOGIA}

Tipo y diseño de investigación: Se ha realizado una investigación básica con diseño correlacional y enfoque transversal (Campbel \& Stanley, 1995).

\section{Muestra}

En la presente investigación se realizó un muestreo no probabilístico de tipo accidental. La muestra estuvo conformada por 2080 alumnas de la Especialidad de Psicología, de Universidades Públicas (691) y Privadas (1380), de las cuales 727 son adolescentes, 1254 jóvenes y 98 adultas, de Lima Metropolitana matriculadas en el año 2019.

\section{Instrumentos}

- Actitudes hacia la Violencia de Género, medidas a través de la Escala de Actitud Hacia la Violencia de Género de Mateos (2011). Son veinte ítems cuyo propósito es obtener valoraciones afectivas sobre "violencia" y "género" en términos de una escala Likert de cinco puntos, desde 1 (totalmente en desacuerdo) hasta 5 (totalmente de acuerdo). La puntuación más elevada, en la Escala significa un mayor rechazo hacia la violencia. Posee dos dimensiones:

- Violencia: Actitud de rechazo hacia los actos violentos y la naturaleza de la violencia en general, sin hacer distinción de género.

- Género: Actitud de rechazo hacia la violencia de cualquier tipo que están dirigidos hacia un género en particular. 
En el presente trabajo hemos estudiado la validez de constructo a través del método del Ítem test, con ítems cuyos $r>0.20$ demostraron su pertinencia. Por otro lado la confiabilidad se obtuvo a través del Alfa de Cronbach obteniendo para la Escala de violencia un $\alpha: 727$ y para la Escala de Género un $\alpha: .848$.

- Escala de Violencia e índice de Severidad, mide la violencia hacia la mujer por parte de la pareja Valdez et al. (2006), está constituida 'por 21 ítems en términos de una escala Likert de cuatro respuestas desde 1 nunca hasta 4 muchas veces. Esta se clasifica en cuatro modalidades:

- Violencia Psicológica: Comportamientos agresivos usados para manipular y controlar a la pareja; manifestados en maltrato verbal, amenazas, aislamiento, desprecio, entre otros.

- Violencia Física: Conductas de abuso con impacto en el cuerpo de la víctima por contacto directo a través golpes, empujones y jalones, o indirecto con limitación de sus movimientos, provocando lesiones usando objetos corto punzantes, armas de fuego y otros.

- Violencia Sexual: Conductas verbales y/o físicas que tienen el objetivo de llevar a cabo una coyunda sexual, pudiendo haber consumación o no de la misma.

- Violencia Económica: Es un tipo de violencia psicológica donde hay abuso en el control de recompensas o castigos de naturaleza crematística.

En cuanto a la validez de constructo, para el presente estudio se obtuvo a través del método del Ítem-test. Todos los ítem fueron $r>0.20$. Con respecto a la confiabilidad se alcanzaron los siguientes coeficientes Alfa de Cronbach: Violencia Psicológica $\alpha .812$;Violencia Física $\alpha .906$; Violencia Sexual $\alpha .840$ y Violencia Económica $\alpha .728$.

\section{Procedimiento}

El manejo y aplicación de los instrumentos psicológicos se concretó previa coordinación con docentes y autoridades de Universidades públicas y privadas en días y horas que fueron programadas. La participación de las estudiantes fue anónima y de manera voluntaria. Previo a los análisis inferenciales se aplicó la prueba KS encontrándose niveles de significancia menores a 0.05 , tanto en las escalas totales, como en las dimensiones, por lo que se utilizaron pruebas no paramétricas.

\section{RESULTADOS}

La tabla 1 da cuenta de una correlación general entre EAVG y el ISV a través de la prueba de Spearman equivalente a -.190 , lo cual indica que es inversa y muy baja. 
Tabla 1

Correlación general de las EAVG y el ISV

\begin{tabular}{lll|r}
\hline & \multicolumn{2}{c}{ ISV } \\
\hline \multirow{3}{*}{ Rho de Spearman } & \multirow{2}{*}{ EAVG } & Coeficiente de correlación &,$- 190^{* *}$ \\
\cline { 3 - 4 } & Sig. (bilateral) &, 000 \\
\cline { 3 - 4 } & $\mathrm{N}$ & 2019 \\
\hline
\end{tabular}

$\mathrm{Al}$ correlacionar las dimensiones de las EAVG y el ISV en la tabla 2 se observa una correlación positiva y alta entre Género y Violencia. Por otro lado, al correlacionar la dimensión género con la violencia psicológica, física, sexual y económica, se observa que estas relaciones son significativas, inversas y bajas. Al correlacionar la dimensión de violencia con la violencia psicológica, física, sexual y económica también se encuentra que son bajas, inversas y significativas. Al correlacionar la violencia psicológica entre la violencia, sexual y económica, estas son entre moderadas y altas, positivas y significativas. La violencia física, al correlacionarse con la violencia sexual y económica muestra relaciones positivas, moderadas y significativas. Al correlacionar la violencia sexual con la violencia económica, esta es alta, positiva y significativa.

Tabla 2

Correlación entre las dimensiones de las EAVG y el ISV

\begin{tabular}{|c|c|c|c|c|c|c|}
\hline \multirow{4}{*}{ GENERO } & & \multicolumn{3}{|c|}{ VIOLENCIA VPSICOLOGICA VFISICA } & \multicolumn{2}{|c|}{ VSEXUAL VECONOMICA } \\
\hline & $\begin{array}{l}\text { Coeficiente de } \\
\text { correlación }\end{array}$ &, $570^{* *}$ &,$- 096^{* *}$ &,$- 184^{* *}$ &,$- 204^{* *}$ &,$- 169^{* *}$ \\
\hline & Sig. (bilateral) & 0.000 & 0.000 & 0.000 & 0.000 & 0.000 \\
\hline & $\mathrm{N}$ & 2050 & 2042 & 2041 & 2037 & 2038 \\
\hline \multirow[t]{3}{*}{ VIOLENCIA } & $\begin{array}{l}\text { Coeficiente de } \\
\text { correlación }\end{array}$ & &,$- 167^{* *}$ &,$- 210^{* *}$ &,$- 224^{* *}$ &,$- 186^{* *}$ \\
\hline & Sig. (bilateral) & & 0.000 & 0.000 & 0.000 & 0.000 \\
\hline & $\mathrm{N}$ & & 2045 & 2045 & 2040 & 2041 \\
\hline \multirow[t]{3}{*}{ VPSICOLOGICA } & $\begin{array}{l}\text { Coeficiente de } \\
\text { correlación }\end{array}$ & & &, $554^{* *}$ &, $407^{* *}$ &, $395^{* *}$ \\
\hline & Sig. (bilateral) & & & 0.000 & 0.000 & 0.000 \\
\hline & $\mathrm{N}$ & & & 2053 & 2049 & 2049 \\
\hline \multirow[t]{3}{*}{ VFISICA } & $\begin{array}{l}\text { Coeficiente de } \\
\text { correlación }\end{array}$ & & & &, $495^{* *}$ &, $467^{* *}$ \\
\hline & Sig. (bilateral) & & & & 0.000 & 0.000 \\
\hline & $\mathrm{N}$ & & & & 2048 & 2048 \\
\hline \multirow[t]{3}{*}{ VSEXUAL } & $\begin{array}{l}\text { Coeficiente de } \\
\text { correlación }\end{array}$ & & & & &, $556^{* *}$ \\
\hline & Sig. (bilateral) & & & & & 0.000 \\
\hline & $\mathrm{N}$ & & & & & 2054 \\
\hline
\end{tabular}

La tabla 3 muestra que no existe diferencia significativa en cuanto a la actitud hacia la violencia de género entre universidades públicas y privadas, mientras que, 
en la variable de severidad de violencia existen diferencias significativas entre universidad pública y privada, siendo esta última la de mayor puntaje rango promedio.

Tabla 3

Diferencias a través de la Prueba de Kruskal Wallis entre las EAVG y el ISV en función al Tipo de Universidad

\begin{tabular}{llrrr}
\hline & \multicolumn{1}{c}{ SD1 } & N & Rango promedio & \multicolumn{1}{c}{ Suma de rangos } \\
\hline \multirow{3}{*}{ EAVGENEROGEN } & Universidad Publica & 684 & 1059,92 & 724988,50 \\
\cline { 2 - 5 } & Universidad Privada & 1366 & 1008,26 & 1377286,50 \\
\cline { 2 - 5 } & Total & 2050 & & \\
\hline \multirow{2}{*}{ ISEVERIDADV } & Universidad Publica & 683 & 980,27 & 669522,50 \\
\cline { 2 - 5 } & Universidad Privada & 1360 & 1042,96 & 1418423,50 \\
\cline { 2 - 5 } & Total & 2043 & & \\
\hline
\end{tabular}

\begin{tabular}{|c|c|c|}
\hline \multicolumn{3}{|c|}{ Estadísticos de prueba ${ }^{a}$} \\
\hline & EAVG & ISV \\
\hline U de Mann-Whitney & 443625,500 & 435936,500 \\
\hline W de Wilcoxon & 1377286,500 & 669522,500 \\
\hline $\mathrm{Z}$ & $-1,867$ & $-2,297$ \\
\hline Sig. asintótica(bilateral) &, 062 & ,022 \\
\hline
\end{tabular}

a. Variable de agrupación: SD1

La tabla 4 da a conocer diferencias significativas entre los rangos de edad (adolescente, joven y adulta) siendo $\mathrm{p}<.05$ para la EAVG. Sin embargo, en el IVS no se observan diferencias significativas entre los grupos etarios.

\section{Tabla 4}

Diferencias a través de la Prueba de Kruskal Wallis entre las EAVG y el ISV en función a la edad

\begin{tabular}{llrr}
\hline & SD2 & N & \multicolumn{1}{c}{ Rango promedio } \\
\hline \multirow{4}{*}{ EAVG } & Adolescentes & 710 & 978,39 \\
\cline { 2 - 4 } & Jovenes & 1242 & 1060,12 \\
\cline { 2 - 4 } & Adultos & 98 & 928,07 \\
\cline { 2 - 4 } ISV & Total & 2050 & 1022,27 \\
\hline \multirow{2}{*}{ Adolescentes } & 709 & 1013,18 \\
\cline { 2 - 4 } & Jovenes & 1236 & 1131,29 \\
\hline
\end{tabular}

\begin{tabular}{lrr}
\multicolumn{4}{c}{ Estadísticos de prueba ${ }^{\mathbf{a}, \mathbf{b}}$} \\
\hline \multicolumn{2}{c}{ EAVG } & ISV \\
\hline H de Kruskal-Wallis & 11,442 & 3,741 \\
\hline gl & 2 & 2 \\
\hline Sig. asintótica &, 003 &, 154 \\
\hline
\end{tabular}

a. Prueba de Kruskal Wallis

b. Variable de agrupación: SD2

La tabla 5 indica que no existen diferencias significativas en la EAVG entre las personas que tienen y no tienen pareja. Mientras que, en el ISV sí existen diferencias significativas entre aquellas personas con pareja y si ella. 
Tabla 5

Diferencias a través de la Prueba de Mann Whitney entre las EAVG y el ISV en función a si tiene o no pareja.

\begin{tabular}{llrrr}
\hline & & N & Rango promedio & \multicolumn{1}{c}{ Suma de rangos } \\
\hline \multirow{3}{*}{ EAVG } & Si tiene pareja & 1018 & 1026,64 & 1045124,50 \\
\cline { 2 - 5 } & No tiene pareja & 1032 & 1024,37 & 1057150,50 \\
\cline { 2 - 5 } & Total & 2050 & & \\
\hline \multirow{3}{*}{ ISV } & Si tiene pareja & 1022 & 1063,58 & 1086977,50 \\
\cline { 2 - 5 } & No tiene pareja & 1021 & 980,38 & 1000968,50 \\
\cline { 2 - 5 } & Total & 2043 & & \\
\hline
\end{tabular}

Estadísticos de prueba

\begin{tabular}{lrr}
\hline & EAVG & \multicolumn{1}{c}{ ISV } \\
\hline U de Mann-Whitney & 524122,500 & 479237,500 \\
\hline W de Wilcoxon & 1057150,500 & 1000968,500 \\
\hline Z &,- 087 & $-3,231$ \\
\hline Sig. asintótica(bilateral) &, 931 &, 001 \\
\hline
\end{tabular}

a. Variable de agrupación: SD4

En la tabla 6 se ve que, en relación al tipo de universidad (pública y privada), las dimensiones de la EAVG y la ISV: género, violencia física, violencia sexual y violencia económica tienen una diferencia significativa. Mientras que en la dimensión violencia y violencia psicológica no existen diferencias significativas.

\section{Tabla 6}

Diferencias entre las dimensiones de EAVG y la ISV en función a la Universidad Pública y Privada

\begin{tabular}{|c|c|c|c|c|}
\hline \multicolumn{5}{|c|}{ Rangos } \\
\hline & SD1 & $\mathbf{N}$ & Rango promedio & Suma de rangos \\
\hline \multirow{3}{*}{ GENERO } & Universidad Publica & 687 & 1071,01 & 735781,00 \\
\hline & Universidad Privada & 1372 & 1009,47 & 1384989,00 \\
\hline & Total & 2059 & & \\
\hline \multirow{3}{*}{ VIOLENCIA } & Universidad Publica & 686 & 1044,66 & 716638,50 \\
\hline & Universidad Privada & 1377 & 1025,69 & 1412377,50 \\
\hline & Total & 2063 & & \\
\hline \multirow{3}{*}{ VPSICOLOGICA } & Universidad Publica & 688 & 997,44 & 686236,50 \\
\hline & Universidad Privada & 1371 & 1046,34 & 1434533,50 \\
\hline & Total & 2059 & & \\
\hline \multirow{3}{*}{ VFISICA } & Universidad Publica & 688 & 976,40 & 671762,50 \\
\hline & Universidad Privada & 1370 & 1056,17 & 1446948,50 \\
\hline & Total & 2058 & & \\
\hline \multirow{3}{*}{ VSEXUAL } & Universidad Publica & 683 & 990,97 & 676830,00 \\
\hline & Universidad Privada & 1371 & 1045,70 & 1433655,00 \\
\hline & Total & 2054 & & \\
\hline \multirow{3}{*}{ VECONOMICA } & Universidad Publica & 684 & 1001,57 & 685072,00 \\
\hline & Universidad Privada & 1372 & 1041,93 & 1429524,00 \\
\hline & Total & 2056 & & \\
\hline
\end{tabular}


Estadísticos de prueba ${ }^{a}$

\begin{tabular}{lrrrrrr}
\hline & GENERO & VIOLENCIA & VPSICOLOGICA & VFISICA & VSEXUAL & VECONOMICA \\
\hline U de Mann-Whitney & 443111,000 & 463624,500 & 449220,500 & 434746,500 & 443244,000 & 450802,000 \\
\hline W de Wilcoxon & 1384989,000 & 1412377,500 & 686236,500 & 671762,500 & 676830,000 & 685072,000 \\
\hline Z & $-2,234$ &,- 686 & $-1,793$ & $-3,676$ & $-3,123$ & $-2,430$ \\
\hline Sig. asintótica(bilateral) &, 025 &, 492 &, 073 &, 000 &, 002 &, 015 \\
\hline
\end{tabular}

a. Variable de agrupación: SD1

En la tabla 7 se observa que existen diferencias entre los grupos etarios con relación a las dimensiones género, violencia y violencia económica, siendo las de mayor rango promedio las Jóvenes para las dimensiones género y violencia de la escala EAVG, y mayor en adultas en la dimensión de violencia económica de la ISV.

\section{Tabla 7}

Diferencias entre las dimensiones de EAVG y la ISV en función a la edad.

\begin{tabular}{|c|c|c|c|}
\hline & & $\mathbf{N}$ & Rango promedio \\
\hline & Jovenes & 1246 & 1050,42 \\
\hline & Adultos & 98 & 892,18 \\
\hline & Total & 2059 & \\
\hline \multirow{4}{*}{ VIOLENCIA } & Adolescentes & 717 & 957,02 \\
\hline & Jovenes & 1247 & 1073,68 \\
\hline & Adultos & 98 & 1039,74 \\
\hline & Total & 2062 & \\
\hline \multirow{4}{*}{ VPSICOLOGICA } & Adolescentes & 714 & 1025,83 \\
\hline & Jovenes & 1247 & 1024,96 \\
\hline & Adultos & 98 & 1124,56 \\
\hline & Total & 2059 & \\
\hline \multirow{4}{*}{ VFISICA } & Adolescentes & 714 & 1032,57 \\
\hline & Jovenes & 1246 & 1026,12 \\
\hline & Adultos & 98 & 1050,12 \\
\hline & Total & 2058 & \\
\hline \multirow{4}{*}{ VSEXUAL } & Adolescentes & 713 & 1020,97 \\
\hline & Jovenes & 1243 & 1024,48 \\
\hline & Adultos & 98 & 1113,36 \\
\hline & Total & 2054 & \\
\hline \multirow{4}{*}{ VECONOMICA } & Adolescentes & 715 & 1012,03 \\
\hline & Jovenes & 1243 & 1028,37 \\
\hline & Adultos & 98 & 1150,29 \\
\hline & Total & 2056 & \\
\hline
\end{tabular}

Estadísticos de prueba ${ }^{\mathrm{a}, \mathrm{b}}$

\begin{tabular}{lrrrrrr}
\hline & GENERO & VIOLENCIA & VPSICOLOGICA & VFISICA & VSEXUAL & VECONOMICA \\
\hline H de Kruskal-Wallis & 7,428 & 17,753 & 2,701 &, 291 & 5,458 & 13,090 \\
\hline Gl & 2 & 2 & 2 & 2 & 2 & 2 \\
\hline Sig. asintótica &, 024 &, 000 &, 259 &, 865 &, 065 &, 001 \\
\hline
\end{tabular}
a. Prueba de Kruskal Wallis
b. Variable de agrupación: SD2 
La tabla 8 señala que existe una diferencia significativa de experiencia de la violencia psicológica y económica entre aquellas personas que tienen pareja y las que no, siendo las que tienen pareja las que presentan un rango promedio superior en ambas dimensiones.

Tabla 8

Diferencias entre las dimensiones de EAVG y la ISV en función a si tienen o no pareja

\begin{tabular}{|c|c|c|c|c|}
\hline & & $\mathbf{N}$ & Rango promedio & Suma de rangos \\
\hline \multirow{3}{*}{ GENERO } & Si tiene pareja & 1022 & 1030,80 & 1053478,00 \\
\hline & No tiene pareja & 1037 & 1029,21 & 1067292,00 \\
\hline & Total & 2059 & & \\
\hline \multirow{3}{*}{ VIOLENCIA } & Si tiene pareja & 1025 & 1031,48 & 1057263,00 \\
\hline & No tiene pareja & 1038 & 1032,52 & 1071753,00 \\
\hline & Total & 2063 & & \\
\hline \multirow{3}{*}{ VPSICOLOGICA } & Si tiene pareja & 1028 & 1072,02 & 1102038,00 \\
\hline & No tiene pareja & 1031 & 988,10 & 1018732,00 \\
\hline & Total & 2059 & & \\
\hline \multirow{3}{*}{ VFISICA } & Si tiene pareja & 1025 & 1037,55 & 1063492,00 \\
\hline & No tiene pareja & 1033 & 1021,51 & 1055219,00 \\
\hline & Total & 2058 & & \\
\hline \multirow{3}{*}{ VSEXUAL } & Si tiene pareja & 1027 & 1041,62 & 1069741,00 \\
\hline & No tiene pareja & 1027 & 1013,38 & 1040744,00 \\
\hline & Total & 2054 & & \\
\hline \multirow{3}{*}{ VECONOMICA } & Si tiene pareja & 1028 & 1047,47 & 1076796,50 \\
\hline & No tiene pareja & 1028 & 1009,53 & 1037799,50 \\
\hline & Total & 2056 & & \\
\hline
\end{tabular}

Estadísticos de prueba ${ }^{a}$

\begin{tabular}{|c|c|c|c|c|c|c|}
\hline & GENERO & VIOLENCIA & SICOLOGICA & VFISICA & VSEXUAL & VECONOMICA \\
\hline U de Mann-Whitney & 529089,000 & 531438,000 & 486736,000 & 521158,000 & 512866,000 & 508893,500 \\
\hline W de Wilcoxon & 1067292,000 & 1057263,000 & 1018732,000 & 1055219,000 & 1040744,000 & 1037799,500 \\
\hline Z &,- 061 &,- 040 & $-3,262$ &,- 784 & $-1,710$ & $-2,424$ \\
\hline Sig. asintótica(bilateral) & ,951 & ,968 & ,001 & ,433 & 087 & 015 \\
\hline
\end{tabular}

a. Variable de agrupación: SD4

La tabla 9 señala que existe una diferencia significativa en la dimensión género de la EAVG, siendo la categoría "autoempleado" el que expresa un menor rango promedio, y las dimensiones violencia psicológica, física, sexual y económica de la ISV, siendo también la categoría "autoempleado" la de mayor rango promedio. 
Tabla 9

Diferencias entre las dimensiones de EAVG y la ISV en función a si tienen o no pareja

\begin{tabular}{|c|c|c|c|c|c|c|}
\hline & & & & $\mathbf{N}$ & & Rango promedio \\
\hline \multirow{4}{*}{ GENERO } & & Empleado & & & 652 & 1000,87 \\
\hline & & Autoempleado & & & 160 & 935,36 \\
\hline & & Desempleado & & & 1247 & 1057,37 \\
\hline & & Total & & & 2059 & \\
\hline \multirow{4}{*}{ VIOLENCIA } & & Empleado & & & 656 & 1051,00 \\
\hline & & Autoempleado & & & 159 & 981,50 \\
\hline & & Desempleado & & & 1248 & 1028,45 \\
\hline & & Total & & & 2063 & \\
\hline \multirow{4}{*}{ VPSICOLOGICA } & & Empleado & & & 658 & 1015,52 \\
\hline & & Autoempleado & & & 160 & 1222,28 \\
\hline & & Desempleado & & & 1241 & 1012,89 \\
\hline & & Total & & & 2059 & \\
\hline \multirow{4}{*}{ VFISICA } & & Empleado & & & 658 & 1035,25 \\
\hline & & Autoempleado & & & 161 & 1123,18 \\
\hline & & Desempleado & & & 1239 & 1014,27 \\
\hline & & Total & & & 2058 & \\
\hline \multirow{4}{*}{ VSEXUAL } & & Empleado & & & 657 & 1025,65 \\
\hline & & Autoempleado & & & 160 & 1143,85 \\
\hline & & Desempleado & & & 1237 & 1013,43 \\
\hline & & Total & & & 2054 & \\
\hline \multirow{4}{*}{ VECONOMICA } & & Empleado & & & 658 & 1024,07 \\
\hline & & Autoempleado & & & 160 & 1157,18 \\
\hline & & Desempleado & & & 1238 & 1014,23 \\
\hline & & Total & & & 2056 & \\
\hline \multicolumn{7}{|c|}{ Estadísticos de prueba ${ }^{a, b}$} \\
\hline & GENERO & VIOLENCIA & VPSICOLOGICA & VFISICA & VSEXUAL & VECONOMICA \\
\hline H de Kruskal-Wallis & 8,409 & 1,881 & 18,831 & 7,985 & 17,233 & 23,155 \\
\hline $\mathrm{gl}$ & 2 & 2 & 2 & 2 & 2 & 2 \\
\hline Sig. asintótica & ,015 & ,390 &, 000 & ,018 & ,000 &, 000 \\
\hline
\end{tabular}

a. Prueba de Kruskal Wallis

b. Variable de agrupación: SD5

\section{DISCUSIÓN}

Respecto al objetivo central de la investigación (relación entre la violencia de género y la probabilidad de riesgo de violencia extrema hacia la pareja), de acuerdo con los resultados conseguidos, se puede verificar que a mayor actitud de rechazo 
a la violencia hay una menor experimentación de violencia de parte de la pareja. La correlación es significativa pero baja, tanto a nivel general como en su análisis dimensional, lo que confirma que estar con una pareja que no agreda habitualmente disminuye el riesgo de ocurrencia de episodios violentos (Pueyo, López y Álvarez, 2008). Asimismo, se coincide en general con los resultados reportados por Aliaga y Figueroa (2019), referentes a correlación entre actitudes sexistas y violencia hacia la mujer en las situaciones de pareja.

Concomitantemente, no hay diferencia en la actitud de rechazo hacia la violencia entre mujeres de universidad públicas y privadas, pero si existe diferencia entre la violencia que estas experimentan, en función a si tienen pareja o no. Puede que haya un mayor número de personas con parejas en las universidades privadas, pero de cualquier manera, como lo señalan Quispe, et al. (2018), el riesgo de que la violencia extrema contra la mujer desemboque finalmente en feminicidio se presenta de preferencia cuando el agresor es desconocido.

También se detectan diferencias en la actitud de rechazo a la violencia de género, mas no a la experimentación de violencia en la relación. Esta actitud de rechazo es mayor en las jóvenes que en las adolescentes y adultas, siendo posible que esté vinculada al hecho de que en las primeras etapas de la vida hay mayor movilidad de cambio de pareja, y eso incrementa la cantidad potencial de decisiones de ruptura que Fernández (2013) indica como la principal fuente o factor desencadenante de problemas vinculados al feminicidio.

No hay diferencia significativa con respecto al rechazo a la violencia a nivel general entre mujeres de universidades públicas y privadas, pero hay menos rechazo a la violencia de género en aquellas mujeres de universidades privadas, estas mismas son las que experimentan mayor violencia física, sexual y económica de parte de sus parejas, con respecto a la violencia psicológica, esta es experimentada por mujeres de ambos tipos de universidad a niveles no diferenciables. Al respecto, Aliaga y Figueroa (2019) ya señalan que la agresión psicológica se presenta con más frecuencia que las demás.

Existen diferencias entre el rechazo a la violencia general y de género entre grupos etarios, siendo las jóvenes las que presentan un mayor rechazo a esta, y las adultas los que presentan una mayor tolerancia. Sin embargo, la violencia psicológica, física y sexual experimentada por las mujeres no tienen diferencias significativas según edad, en contraste con la violencia económica, la cual es experimentada en mayor medida por las adultas, seguida por las jóvenes.

No hay diferencia de actitud de rechazo a la violencia general y de género entre las personas que tienen pareja y las que no. Sin embargo, hay diferencia entre la violencia psicológica y económica que experimentan, siendo aquellas que tienen pareja las que sufren en mayor medida de estas conductas. También existen 
diferencias significativas en la violencia psicológica, sexual, física y económica entre las personas según su situación laboral, siendo las personas auto empleadas las que más sufren de violencia, estas mismas son las que presentan un menor rechazo a la violencia de género. Las mujeres desempleadas presentan el mayor rechazo a la violencia de género, así como ser las que experimentan menor violencia. Evidentemente, este mayor rechazo es lógico si se considera la también mayor vulnerabilidad económica de esas personas y la connotación de seres subordinados a otros (los hombres) que conlleva (Montenegro, 2018). Sin embargo, con respecto a la violencia general, no existen diferencias en la actitud de rechazo entre ningún grupo.

\section{CONCLUSIONES}

- En las estudiantes universitarias, a mayor actitud de rechazo a la violencia, menor experimentación de violencia.

- Las mujeres de las universidades privadas experimentan mayor violencia física, sexual y económica que las de las universidades públicas, así como también presentan un menor rechazo a la violencia de género.

- Las jóvenes presentan un mayor rechazo a la violencia, y las adultas son los que más la aceptan, sin embargo, todos los grupos etarios experimentan el mismo nivel de violencia física, psicológica y sexual.

- Las mujeres universitarias que tienen pareja y las que no, rechazan en igual medida la violencia, pero son las primeras de estas las que experimentan mayor violencia psicológica y económica.

- Los instrumentos seleccionados, adaptados a nuestra realidad para evaluar la actitud hacia la violencia y la violencia muestran ser confiables

- Por último, las personas auto empleadas son las que sufren mayor violencia en todos sus tipos a comparación de aquellas empleadas y desempleadas, son también las que menos rechazan a la violencia de género. Todas estas rechazan en igual medida la violencia general.

\section{RECOMENDACIONES}

- Es importante llevar a cabo programas de concientización sobre la violencia de género en mujeres de universidades privadas.

- Se deben diseñar estudios controlando el número de mujeres que cuentan y no cuentan con pareja, para determinar la influencia de la variable tipo de universidad en los resultados de actitud hacia la violencia y violencia experimentada. 
- En necesario seguir estudiando la variedad de aspectos sociopolíticos y económicos que probabilizan la relación entre violencia experimentada y el tipo de universidad de procedencia.

\section{Agradecimientos / Acknowledgments:}

Se agradece el apoyo brindado por los las autoridades de las universidades públicas y privadas que participaron en este estudio.

\section{Fuentes de financiamiento / Funding:}

Financiado por la Universidad Nacional Mayor de San Marcos. Proyecto con código A19180171, aprobado con Resolución Rectoral 03556-R-19

\section{Rol de los autores / Authors Roles:}

CVC: Responsable de la investigación.

AGM: Corresponsable de la investigación, análisis de datos.

WMU: Responsable del marco teórico.

VML: Responsable de los antecedentes.

RPV : Análisis de datos.

JVS : Redacción del Articulo.

SCE : Presentación de resultados.

DZF: Administración de instrumentos.

KPL: Administración de los instrumentos.

SFG: Responsable de la recogida de datos.

\section{Aspectos éticos / legales; Ethics / legals:}

Los autores declaran no haber incurrido en aspectos antiéticos respetando los códigos de investigación con humanos, se solicitó la autorización respectiva y los participantes firmaron el consentimiento informado el cual brindaba datos de la naturaleza, medios y objetivos de la investigación.

\section{Conflicto de intereses / Competing interests:}

Los autores declaran no haber presentado conflicto de intereses en la investigación y elaboración del artículo. 


\section{REFERENCIAS}

Abramovich, E. (2014). Historia de la sexualidad en occidente. En A. M. Vega (Comp.). Sexualidades. Guía de conceptos y herramientas para aprender, vivir y compartir (pp. 17-28). Buenos Aires: Lugar Editorial.

Aliaga, M. y Figueroa, L. (2019). Violencia de pareja hacia la mujer y actitudes sexistas en universitarios (Tesis de pregrado). Universidad Privada Antonio Guillermo Urrelo, Cajamarca.

América Noticias (27/12/2019). Feminicidios en Perú 2019: Se registran 164 casos entre enero y diciembre. Recuperado de: https://www.americatv.com.pe/noticias/actualidad/feminicidios-2019-uno-uno-crimenes-contra-mujeres-n353912

Arcila, A. (2014). Comportamiento sexual: Entre la biología, la moral y el derecho. Manizales: Universidad de Caldas.

Bandura (1984). Análisis del aprendizaje social de la agresión. En A. Bandura y E. Ribes (Eds.). Modificación de conducta: Análisis de la agresión y la delincuencia (pp. 307347). México: Trillas, Orig. 1975.

Beaducel, A., \& Herzberg, P. Y. (2006). On the performance of maximum likelihood versus means and variance adjusted weighted least squares estimation in CFA. Structural Equation Modeling, 13, 186-203. doi: 10.1207/s15328007sem1302_2.

Campbel, D. \& Stanley, J. (1995). Diseños experimentales y cuasiexperimentales en la investigación social. Buenos aires: Amorrortu.

Connel, R. \& Pearse, R. (2015). Gênero, uma perspectiva global. São Paulo: Versos.

Cunha, C. R., Alves, A. C., Gushi, H. A., Farias, K. F., Murrins, R. M., Moraes, L., Viera, M., Ferreira, R. \& Barreto, R. A.. (2013), Relaçðes afetivas e cultura: A influência do ideal de amor sobre os relacionamentos reais. Em: C. Zeglio I Finotelli Jr. \& O. Rodrigues (Orgs.). Relaçðes conjugais. Discutindo alternativas para melhor qualidade de vida (pp. 117-122). Säo Paulo: Zagodoni Editora.

Fernández J. (2013). Riesgo de feminicidio de género en situaciones de ruptura de la relación de pareja. Estudios Penales y Criminológicos, XXXIII, ISSN 1137-7550, №. 33, 2013, págs. 149-173

Gómez, J. (2013). Psicología de la sexualidad. Madrid: Alianza.

Hernández, W., Raguz, M., Morales, H. y Burga, A. (2018). Feminicidio: Determinantes y evaluación del riesgo. Informe Final. Lima: Universidad de Lima.

Hernández, W. (2019, 14 de enero). Violencia con riesgo de feminicidio. Lima: Diario El Comercio.

Matamoros, F. G. (2014). El agresor sexual. México: Trillas.

Mateos, A. (2011) Necesidades socioeducativas en la adolescencia sobre la violencia de género: Propuesta educativa. Tesis Doctoral de la facultad de Pedagogía. Universidad de Barcelona. 
Ministerio de la Mujer y Poblaciones Vulnerables del Perú (2016). Violencia basada en género. Marco conceptual para las políticas públicas y la acción del estado. Lima: Autor.

Montenegro, J. (2018). Estado del arte sobre el feminicidio en el Perú. Características y tendencias. Revista Científica Paian, 9 (2), 17-34.

Organización de las Naciones Unidas (1993). Declaración sobre la eliminación de la violencia contra la mujer. New York. Recuperado de: https://www.ohchr.org/sp/professionalinterest/pages/violenceagainstwomen.aspx

Organización Mundial de la Salud (2002). Informe Mundial de la Violencia y de la Salud: Resumen. Washington, DC: Organización Panamericana de la Salud.

Organización Mundial de la Salud (2014). Respuesta a la violencia de pareja y a la violencia sexual contra las mujeres. Directrices de la OMS para la práctica clínica y las politicas. Washington, DC: Organización Panamericana de la Salud.

Observatorio Nacional de la Violencia contra las Mujeres y los Integrantes del Grupo Familiar (visto: 2019). Mecanismo de Seguimiento del Plan Nacional Contra la Violencia de Género 2016-2021. Recuperado de: https://observatorioviolencia.pe/plannacional/plan-nacional-contra-la-violencia-de-genero-2016-2021/

Pacheco, M. (2015). Actitud hacia la violencia contra la mujer en la relación de pareja y clima social familiar en adolescentes. Interacciones. Revista de Avances en Psicología, 1(1), 29-44. https://doi.org/10.24016/2015.v1n1.2

Pueyo, A. A., López, S. y Álvarez, E. (2008). Valoración del riesgo de violencia contra la pareja por medio de la SARA. Papeles del Psicólogo, 29(1), 107-122.

Quintana Peña, A., Grajeda Montalvo, A., Malaver Soto, C., Medina Curi, N., Montgomery Urday, W., Ruiz Sánchez, G., \& Ojeda Mercado, G. (2020). Estereotipos de género y violencia encubierta de pareja en hombres y mujeres de 18 a 24 años. $R e-$ vista De Investigación En Psicología, 22(2), 181-196. https://doi.org/10.15381/rinvp. v22i2.17420

Quispe, M., Curro O., Córdova M., Pastor N., Puza G. y Oyola A. (2018). Violencia extrema contra la mujer y feminicidio en el Perú. Revista Cubana de Salud Pública, ISSN 0864-3466versión On-line ISSN 1561-3127, 44(2), 278-294.

Rodríguez-Miñón, E. (2016). Factores de riesgo o vulnerabilidad ante la violencia de género de las mujeres residentes en municipios pequeños o rurales desde una perspectiva ecológica. Máster Universitario en igualdad y género en el ámbito público y privado. Barcelona: Universitat Jaume I.

Russell, D. A. \& Harmes, R. A. (2006). Feminicidio: Una perspectiva global. México: Universidad Autónoma de México. 